\title{
The Chemical Mechanism of Myosin-I: Implications for Actin-based Motility and the Evolution of the Myosin Family of Motor Proteins
}

\author{
Thomas D. Pollard and E. Michael Ostap \\ Department of Cell Biology and Anatomy, The Johns Hopkins University School of Medicine, Baltimore, MD, \\ 21205
}

Key words: myosin/actin/ATP/motility

\begin{abstract}
The Acanthamoeba myosin-IA and myosin-IB molecular motors bind to membranes, so they may produce the force to move organelles and membranes along actin filaments. We have determined the rate constants for the actin-activated myosin-I ATPase by pre-steady state kinetic analysis. ATP binds rapidly to myosin-I and dissociates the enzyme from actin filaments at a rate $>500 \mathrm{~s}^{-1}$. Myosin-I hydrolyzes ATP to ADP and inorganic phosphate $\left(P_{\mathrm{i}}\right)$ at $20-50 \mathrm{~s}^{-1}$. Phosphate dissociation is the rate limiting step in the ATPase cycle, $0.01 \mathrm{~s}^{-1}$ for myosin-I alone and at $10 \mathrm{~s}^{-1}$ when myosin-I is bound to actin filaments. ADP dissociation is rapid. Phosphorylation controls the ATPase cycle by increasing the rate of phosphate release from myosin-I bound to

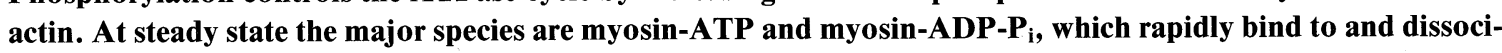
ate from actin filaments. During the ATPase cycle myosin-I binds so weakly to actin filaments that it cannot support processive movement like kinesin, unless several motors cluster together on a membrane or actin filament. These properties of the enzyme emphasize the importance of characterizing mechanisms that promote the selfassociation of myosin-I isoforms at specific binding sites in cells.
\end{abstract}

The discovery of Acanthamoeba myosin-I (20) was the first indication that the myosin family of motor proteins includes members significantly different from muscle myosin. Now we recognize more than ten classes of myosin, all with motor domains that use ATP hydrolysis to generate the force for movement along actin filaments and tail domains that specialize them for unique functions in cells (8).

Myosin-I's have several functionally distinct domains (Fig. 1) (22). The N-terminal 690 residues are homologous with the catalytic head domain of muscle myosinII, with the exception that myosin-I's lack the first 80 residues that form a six-stranded antiparallel beta-sheet (23). The head domain has ATPase activity that is stimulated by its binding to actin filaments. Many myosin-I isoforms have one or more IQ domains that are believed to bind light chains (28). In vertebrate myosin-I's these light chains include calmodulin. The Acanthamoeba myosin-I light chains differ from both calmodulin and myosin-II light chains (P. Matsudaira, personal communication). The C-terminal tail region of myosinIs set them apart from other myosins. None of the myosin-Is have the heptad repeats characteristic of the alpha-helical coiled-coil tails of myosin-II and some other myosins. Although the sequences of the myosin-I

Phone: 410-955-5664, FAX: 410-955-4129

e-mail: pollard@salk.edu tails are themselves very diverse, they do have some common structural elements that may be related to their function (22). Acanthamoeba myosin-I tails contain (1) a region of high positive charge that accounts for the ability of myosin-I to bind to acidic phospholipids such as phosphatidylserine (9), (2) a region rich in glycine, proline, and alanine/serine residues that may bind to actin $(9,16,17)$ and $(3)$ an SH3 domain that may be important for the specific cellular localization of the myosin-I isoforms.

Each of the three myosin-I isoforms identified in Acanthamoeba has a distinctive distribution: myosinIA is largely cytoplasmic, myosin-IB is concentrated on the plasma membrane, and myosin-IC also binds the plasma membrane and is the only known isoform associated with the contractile vacuole $(3,4,29)$. Loading

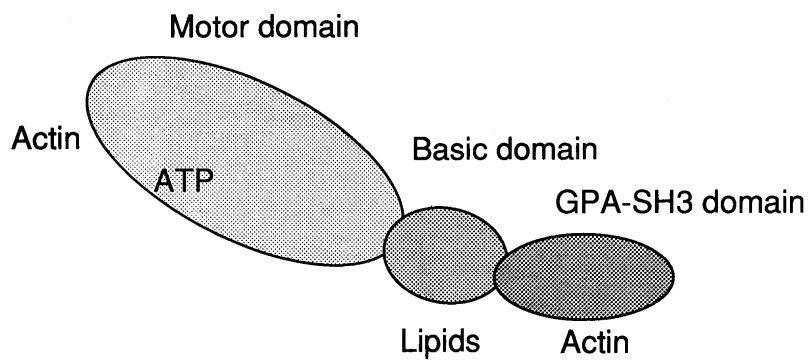

Fig. 1. Schematic drawing of the domains of myosin-I. 
amoebas with antibodies that inhibit myosin-IC compromises the function of the contractile vacuole, so that the cells lyse in dilute media (10), conditions similar to the osmotic challenge that a cell would encounter during a rain storm. Antibodies to other myosin-I isoforms did not inhibit the contractile vacuole. These antibody inhibition experiments and the localization work suggests that each myosin-I isoform has specific functions that have provided the amoebas with a selective advantage during evolution. These functions may be difficult to appreciate unless one challenges the cell with extreme, but naturally occurring conditions. For example, disruption of one to three of the myosin-I genes in Dictyostelium $(15,26)$ is not lethal under laboratory conditions, although the cells become progressively more disabled for motility and endocytosis as additional genes are knocked out.

Steady state enzyme assays $(1,2,17,21)$ provided the background for our analysis of the mechanism of myosin-I by transient state kinetics (19). Like muscle myosin, myosin-I turns over ATP very slowly at a steady state rate of about $0.01 \mathrm{~s}^{-1}$. In the presence of a cofactor protein, actin filaments stimulate the steady state ATPase rate by $>100$ fold to $10 \mathrm{~s}^{-1}(21)$. The cofactor is a heavy chain kinase (18), which phosphorylates a single serine or threonine residue (depending upon the myosin-I isoform) located in the sequence at a position corresponding to residue 411 of chicken muscle myosin $(5,6)$. Phosphatidylserine and autophosphorylation stimulate the activity of the heavy chain kinase (7).

Like other myosins, myosin-I binds tightly to actin filaments in the absence of ATP. In the presence of ATP, the myosin-I head binds weakly to actin filaments, but this weak interaction stimulates the ATPase activity and drives the gliding of actin filaments over substrates (nitrocellulose or phosphatidylserine in lipid bilayers supported on glass) that bind myosin (30).

To learn how myosin-I uses ATP to produce movements of actin filaments, we determined the mechanism

Table I. Summary of Myosin-IA and Myosin-IB Rate AND EQUILIBRIUM CoNSTANTS*

\begin{tabular}{lcc}
\hline Rate Constant & MIA & MIB \\
\hline $\mathrm{K}_{1} \mathrm{k}_{2}$ & $0.74 \mu \mathrm{M}^{-1} \mathrm{~s}^{-1}$ & $3.37 \mu \mathrm{M}^{-1} \mathrm{~s}^{-1}$ \\
$\mathrm{~K}_{1}^{\prime} \mathrm{k}_{2}^{\prime}$ & $0.29 \mu \mathrm{M}^{-1} \mathrm{~s}^{-1}$ & $1.3 \mu \mathrm{M}^{-1} \mathrm{~s}^{-1}$ \\
$\mathrm{k}_{2}{ }^{\prime}$ & $>300 \mathrm{~s}^{-1}$ & $>300 \mathrm{~s}^{-1}$ \\
$\mathrm{~K}_{+3}+\mathrm{k}_{-3}$ & $\geq 16 \mathrm{~s}^{-1}$ & $\geq 53 \mathrm{~s}^{-1}$ \\
$\mathrm{~K}_{3}$ & 1.9 & 1.5 \\
$\mathrm{k}_{5}{ }^{\prime}$ & $\mathrm{N} . \mathrm{D}$. & $>80 \mathrm{~s}^{-1}$ \\
$\mathrm{~K}_{5}{ }^{\prime}$ & $1.1 \times 10^{4} \mathrm{M}^{-1}$ & $1.9 \times 10^{4} \mathrm{M}^{-1}$ \\
$\mathrm{~K}_{6}$ & $1.7 \times 10^{7} \mathrm{M}^{-1}$ & $2.0 \times 10^{7} \mathrm{M}^{-1}$ \\
\hline
\end{tabular}

* Phosphorylation of the myosin-I heavy-chain does not affect these rate constants. of ATP hydrolysis by Acanthamoeba myosin-IA (MIA) and myosin-IB (MIB) by transient kinetic techniques. The result is a set of rate and equilibrium constants (Table I, Figure 2) that determine what kind of movements that the enzyme can produce and how the enzyme is controlled. The enzyme mechanisms of these myosinI's from a protozoa are remarkably similar to the mechanism of vertebrate skeletal muscle myosin-II, the only other member of the myosin superfamily that has been analyzed in this detail.

\section{Analysis of the ATPase Mechanism}

We analyzed the kinetics of myosin-IA and myosinIB purified from Acanthamoeba castellanii using stopped-flow and quenched-flow methods. Fluorescent analogs of actin (12) and ATP (14) provided sensitive signals for these assays. To learn how heavy chain phosphorylation controls the enzyme activity, we compared unphosphorylated myosin-I with myosin-I that was phosphorylated with myosin-I heavy chain kinase. Phosphorylated myosin-I was repurified to remove the contaminating kinase and nucleotide. Actin was from rabbit skeletal muscle.

The maximum steady-state ATPase rates of phosphorylated MIA and MIB in low ionic strength buffer were achieved with high concentrations of actin. These rates are $7 \mathrm{~s}^{-1}$ for myosin-IA and $6 \mathrm{~s}^{-1}$ for myosin-IB. These are the values of the rate limiting steps in the mechanisms of these two enzymes.

Experiment One: Mant-ATP Binding to Myosin-I. Mixing myosin-I with methylanthraniloyl ATP (mantATP; 14) produces an increase in fluorescence with an exponential time course. The observed rate constants are proportional to the concentration of the ATP ana$\log$ over the range tested, up to rates of $30 \mathrm{~s}^{-1}$. The slopes of these plots give the apparent second order rate constants for nucleotide binding of $0.7 \mu \mathrm{M}^{-1} \mathrm{~s}^{-1}$ for MIA and 3.4 $\mu \mathrm{M}^{-1} \mathrm{~S}^{-1}$ for MIB with or without heavy chain phosphorylation (Table I). These apparent second order rate constants for mant-ATP binding are actually the product of the equilibrium constant for ATP binding and the first order rate constant for the isomerization of the myosin which produces the fluorescence change in the bound nucleotide. Under the conditions tested, the isomerization step is faster than the binding step, so the experiment measures the rate of the binding step and places a lower limit on the rate of the isomerization step.

Under physiological conditions with $1 \mathrm{mM}$ ATP, the rate of ATP binding is very fast, $700 \mathrm{~s}^{-1}$ for myosin-IA and $>3,000 \mathrm{~s}^{-1}$ for myosin-IB. This experiment places a lower bound of $30 \mathrm{~s}^{-1}$ on the rate of the isomerization. Thus, neither ATP binding nor the conformational change is rate limiting in the ATPase cycle or regulated by heavy chain phosphorylation. 


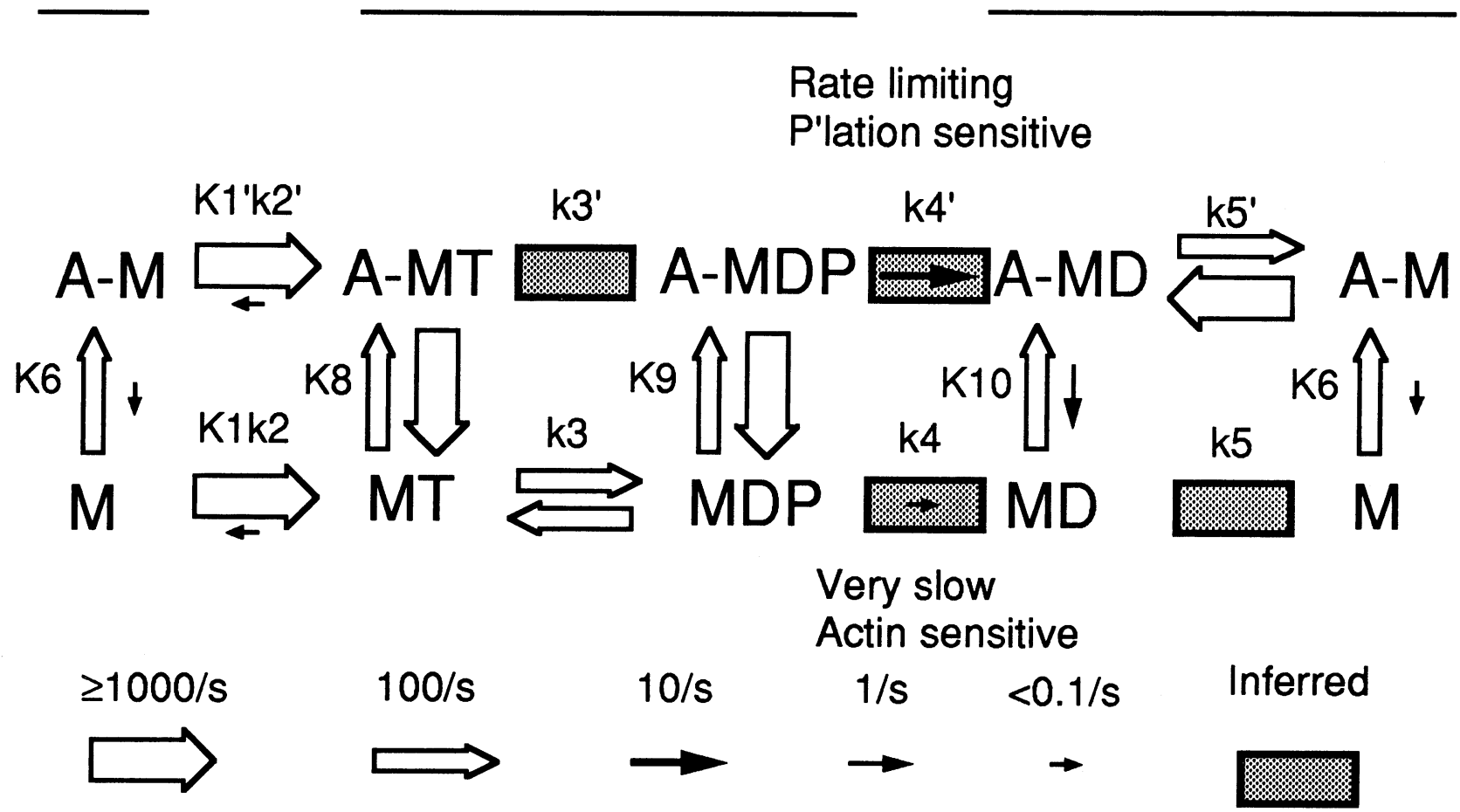

Fig. 2. Chemical mechanism of myosin-I. $A=$ actin filaments, $M=$ myosin-I, $T=A T P, D=A D P, P=$ inorganic phosphate. The sizes of the arrows indicate the approximate vaules of the rate constants.

Experiment Two: Myosin-I Binding to Pyrene-Actin. Myosin-I binding quenches the fluorescence of pyrenelabeled actin filaments by $50-60 \%(12,24)$, providing an assay to measure association and dissociation of the actomyosin-I complex. The dissociation equilibrium constants for actin filament binding $\left(1 / \mathrm{K}_{6}\right)$ in the absence of ATP are $0.06 \mu \mathrm{M}$ for MIA and $0.05 \mu \mathrm{M}$ for MIB (Table I). These values are similar to skeletal muscle and Dictyostelium myosin-II binding to actin under the same conditions (24).

Mixing ATP with myosin-IA or -IB bound to pyrenelabeled actin filaments produces an increase in fluorescence to the level of actin alone. The time course of the increase in fluorescence is exponential and depends on the concentration of ATP up to the highest rates $\left(>300 \mathrm{~s}^{-1}\right)$ that we could measure within the limits of protein concentrations and instrumental dead time. Heavy chain phosphorylation did not affect the rates of actomyosin-I dissociation by ATP.

The dissociation reaction includes at least three steps: (1) rapid equilibrium binding of ATP; (2) a rate limiting conformational change in the myosin induced by ATPbinding; and (3) rapid dissociation of the proteins. The linear dependence of the observed rate constants for myosin-I dissociation give apparent second order rate constants $\left(\mathrm{K}_{1}{ }^{\prime} \mathrm{k}_{2}{ }^{\prime}\right)$ of $0.3 \mu \mathrm{M}^{-1} \mathrm{~s}^{-1}$ for MIA and 1.3 $\mu \mathrm{M}^{-1} \mathrm{~S}^{-1}$ for MIB (Table I). These apparent second order rate constants are similar to those for mant-ATP binding (Table I), since the dissociation reaction is faster than the ATP binding and isomerization steps.

From this experiment, we draw several conclusions. First, ATP dissociates the MIA and MIB heads completely from the low concentrations of actin filaments allowed by the experimental design. Second, the binding of the ATP-insensitive site on the myosin-I tail does not affect the fluorescence of pyrene-labeled actin. Third, dissociation of myosin-I from actin filaments is not the rate limiting step in the ATPase cycle in the presence of physiological concentrations of ATP. Fourth, heavy chain phosphorylation does not regulate actomyosin dissociation.

Experiment Three: ATP hydrolysis. We used rapid quench experiments to measure the rate of ATP hydrolysis and the equilibrium constant for the reaction. We measured the time course of ATP hydrolysis by quenching the reaction at different time points with acid. We also measured the concentration of myosin-I heads that bind ATP irreversibly by quenching the reaction with unlabeled ATP. These reactions include several steps: rapid ATP binding $\left(\mathrm{K}_{1}\right)$ and the conformational change $\left(\mathrm{k}_{2}\right)$ (already measured in the mant-ATP binding experiment), followed by ATP hydrolysis to ADP and inor- 
ganic phosphate $\left(\mathrm{P}_{\mathrm{i}}\right)$ and then the dissociation of these products.

Mixing ATP with MIA or MIB produces a rapid burst of ATP hydrolysis with an exponential time course, followed by a slow phase that looks like a plateau on the rapid time-scale of the experiment. The existence of the burst phase indicates that the rate limiting step occurs after the hydrolysis of ATP. The time course of the phosphate burst measures the sum of the rate constants for reversible ATP hydrolysis $\left(\mathrm{k}_{+3}+\right.$ $\mathrm{k}_{-3}$ ). The curves fit single exponentials with rates of 16 $\mathrm{s}^{-1}$ for MIA and $53 \mathrm{~s}^{-1}$ for MIB in $25 \mu \mathrm{M}$ ATP (Table I). These are lower limits of the hydrolysis rate constants, because the low ATP concentrations that we could use in these experiments limits the rate of hydrolysis. Even these minimal hydrolysis rates exceed the maximum steady state turn over rate at high actin concentrations, so hydrolysis is not the rate limiting step for the ATPase cycle.

The cold-chase experiments gave apparent second order rate constants for ATP binding $\left(\mathrm{K}_{1} \mathrm{k}_{2}\right)$ that agree very well with the apparent second-order rate constants determined by the mant-ATP stopped-flow experiments. Comparison of the acid quench and cold-chase experiments, gave the amplitudes of the phosphate bursts and allowed calculation of the equilibrium constants for ATP hydrolysis $\left(\mathrm{K}_{3}\right)$ of 1.9 for MIA and 1.5 MIB (Table I).

From this experiment we conclude that ATP hydrolysis is a rapid equilibrium with an equilibrium constant near one. Hydrolysis is not rate limiting in the ATPase cycle. Since the rate limiting step follows hydrolysis and since ATP dissociates myosin-I completely from actin, we can also conclude that the myosin-I-ADP-P com- $_{\mathrm{i}}$ plex binds weakly to actin filaments like myosin-I-ATP.

Experiment Four: ADP Binding and Dissociation. Myosin-I with bound ADP binds relatively strongly to pyrene-labeled actin filaments and quenches the fluorescence. This made it possible to measure the affinity of the actomyosin-I complex for ADP. Dissociation of ADP limits the rate of ATP binding and the subsequent rapid dissociation of the actomyosin-I complex, which we monitored by the fluorescence of pyrene-labeled actin. The dissociation equilibrium constants for ADP are $93 \mu \mathrm{M}$ for MIA and $53 \mu \mathrm{M}$ for MIB (Table I). The rate constant for the dissociation of ADP is $84 \mathrm{~s}^{-1}$. From this experiment we conclude that ADP binds rapidly and reversibly to the actomyosin-I complex but does not dissociate it like ATP. ADP dissociation from the actomyosin-I complex is not rate limiting in the ATPase cycle.

\section{Interpretation}

The kinetic experiments provide enough information to propose a complete chemical mechanism for the acto-
myosin-I ATPase cycle (Fig. 2). With physiological concentrations of ATP, rates of all the measured reactions would be faster than the steady-state ATPase rate, so one or more steps between ATP hydrolysis and ADP release is rate-limiting in the ATPase cycle. The most likely candidates for this slow step are phosphate release $\left(\mathrm{k}_{4}{ }^{\prime}\right)$, or an isomerization preceding phosphate release (Fig. 2). Phosphate release has long been believed to regulate the transition between the weak-binding (nonforce-bearing) and strong-binding (force-bearing) states in the skeletal muscle myosin-II ATPase cycle (11).

Given this rate limiting step, the predominant steadystate intermediates will be M.ATP and M.ADP.P $P_{i}$ both in rapid equilibria between their free and actin-bound states (Fig. 2). Their weak binding to actin filaments reflects the fact that these intermediates dissociate rapidly nearly every time that they bind to actin filaments, just like the corresponding intermediates of muscle myosin. Although their interactions with actin are transient, the actin stimulates the dissociation of $P_{i}$ from the AM.DP intermediate. Consequently, the pathway out of the four-way rapid equilibrium will be by this reaction. This transition is generally regarded to be the force producing step, because of the large free energy change (27). The two intermediates that follow $P_{i}$ release, AMD and AM, both bind tightly to actin filaments, allowing the force produced by $\mathrm{P}_{\mathrm{i}}$ release to be sustained until ATP rebinds to AM and reestablishes the rapid four-way equilibrium of weakly bound state (Fig. 2). This mechanism is remarkably similar to that of muscle of muscle myosin.

\section{Regulation of Myosin-I by Heavy-Chain Phosphoryla- tion}

Heavy chain phosphorylation regulates the myosin-I by increasing the actin-activated ATPase rate $>20$ fold $(2,21)$. Phosphorylation does not alter the rate of any of the steps that we measured or the affinity of MIA to actin in the presence of ATP (17). Thus phosphorylation must exert its effect at the rate limiting step between ATP hydrolysis and ADP release, either $P_{i}$ release itself or a conformations change that precedes $P_{i}$ release (Fig. 2 ). It is fascinating that the phosphorylated serine and threonine residues of myosin-I correspond to a glutamic acid in the actin binding site of skeletal muscle myo$\sin$. Thus phosphorylation of myosin-I alters its charge to resemble muscle myosin.

\section{Myosin-I Function In Vivo}

The ATPase mechanism tells us that myosin-I spends most of the time during the ATPase cycle weakly bound to, or detached from, the actin filament. Therefore, one myosin-I molecule could not support processive movement of molecular cargo along an actin filament like kinesin moves along a microtubule $(13,25)$. Instead, sever- 
al myosin-I molecules must cluster together, perhaps on a membrane to support processive motility. Muscle myosin forms bipolar filaments with several hundred myosin molecules, assuring that many heads are always attached to an actin filament even though each individual head dances rapidly on and off of the filament.

\section{Evolution of Myosin}

We find it remarkable that the ATPase mechanisms of Acanthamoeba myosin-I and vertebrate skeletal muscle myosin are so similar, since the amoeba protein is thought to participate in membrane movements, while the vertebrate enzyme powers the one dimensional sliding of filaments which contracts muscle. The resemblance of their chemical mechanisms suggests that the basic force producing process evolved more than one billion years ago, at the time that this protozoan and vertebrate animals had a common ancestor. This force producing strategy with predominant weak-binding intermediates preceding short-lived tightly-bound states, has been conserved ever since. Not every myosin will be as similar as these first two fully characterized examples, but their properties suggest taht some fundamental aspects of the energy transducing process must provide a selective advantage for cells which put quite different demands on their actomyosin systems.

\section{REFERENCES}

1. Albanesi, J.P., Fujisaki, H., and Korn, E.D. 1985. A kinetic model for the molecular basis of the contractile activity of Acanthamoeba myosin-IA and myosin-IB. J. Biol. Chem., 260: 11174-11179.

2. Albanesi, J.P., Hammer, J.A., and Korn, E.D. 1983. The interaction of F-actin with phosphorylated and unphosphorylated myosin-IA and myosin-IB from Acanthamoeba castellanii. $J$. Biol. Chem., 258: 176-181.

3. BAINES, I.C., BRZESKA, H., and Korn, E.D. 1992. Differential localization of Acanthamoeba myosin-I isoforms. J. Cell Biol., 119: 1193-1203.

4. Baines, I.C., Corigliano-Murphy, A., and Korn, E.D. 1995. Quantification and localization of phosphorylated myosin-I isoforms in Acanthamoeba castellanii. J. Cell Biol., 130: 591-603.

5. Bement, W.M. and Mooseker, M.S. 1995. TEDS Rule: A molecular rationale for differential regulation of myosins by phosphorylation of the heavy chain head. Cell Motil. Cytoskeleton, 31: 87-92.

6. Brzeska, H., Lynch, T.J., Martin, B., and Korn, E.D. 1989. The localization and sequences of the phosphorylation sites of Acanthamoeba myosin-I. An improved method for locating the phosphorylated amino acid. J. Biol. Chem., 264: 19340-19348.

7. BrzesKa, H., LyNCh, T.J., and Korn, E.D. 1990. Acanthamoeba myosin-I heavy-chain kinase is activated by phosphatidylserine-enchanced phosphorylation. J. Biol. Chem., 265: 35913594.

8. Cheney, R.E., Riley, M.A., and Mooseker, M.S. 1993.
Phylogenetic analysis of the myosin superfamily. Cell Motil. Cytoskeleton, 24: 215-223.

9. Doberstein, S.K. and Pollard, T.D. 1992. Localization and specificity of the phospholipid and actin-binding sites on the tail of Acanthamoeba myosin-IC. J. Cell Biol., 117: 1241-1249.

10. Doberstein, S.K., Baines, I.C., Weigand, G., Korn, E.D., and Pollard, T.D. 1993. Inhibition of contractile vacuole function in vivo by antibodies against myosin-I. Nature (Lond.), 365: 841-843.

11. Eisenberg, E. and Greene, L.E. 1980. The relation of muscle biochemistry to muscle physiology. Annu. Rev. Physiol., 42: 293-309.

12. GeEves, M.A. and JefFries, T.E. 1988. The effect of nucleotide upon a specific isomerization of actomyosin subfragment 1 . Biochem J., 256: 41-46.

13. Gilbert, S.P., Webb, M.R., Brune, M., and Johnson, K.A. 1995. Pathway of processive ATP hydrolysis by kinesin. Nature (Lond.), 373: 671-676.

14. Hiratsuka, T. 1983. New ribose-modifies fluorescent analogs of adenine and guanine nucleotides available as substrates for various enzymes. Biochim. Biophys. Acta, 742: 496-508.

15. Jung, G., Fukui, Y., Martin, B., and Hammer III, J.A. 1993. Sequence, expression pattern, intracellular localization, and targeted disruption of the Dictyostelium myosin ID heavy chain isoform. J. Cell Biol., 268: 14981-14990.

16. Jung, G. and Hammer III, J.A. 1994. The actin binding site in the tail domain of Dictyostelium myosin IC (myoC) resides within the glycine- and proline-rich sequence (tail homology 2). FEBS Lett., 342: 197-202.

17. Lynch, T.J., Albanesi, J.P., Korn, E.D., Robinson, E.A., Bowers, B., and FuJISAKI, H. 1986. ATPase activities and actin-binding properties of subfragments of Acanthamoeba myosin IA. J. Biol. Chem., 261: 17156-17162.

18. MarUtA, H. and Korn, E.D. 1977. Acanthamoeba cofactor protein is a heavy chain kinase required for actin activation of the $\mathrm{Mg}^{2+}$-ATPase activity of Acanthamoeba myosin-I. J. Biol. Chem., 252: 8329-8332.

19. Ostap, E.M. and Pollard, T.D. 1996. Biochemical kinetic characterization of the Acanthamoeba myosin-I ATPase. $J$. Cell Biol., 132: 1053-1060.

20. Pollard, T.D. and Korn, E.D. 1973a. Acanthamoeba myosin-I. Isolation from Acanthamoeba castellanii of an enzyme similar to muscle myosin. J. Biol. Chem., 248: 4682-4690.

21. Pollard, T.D. and Korn, E.D. 1973b. Acanthamoeba myosin. II. Interaction with actin and with a new cofactor protein required for actin activation of $\mathrm{Mg}^{++}$ATPase activity. J. Biol. Chem., 248: 4691-4697.

22. Pollard, T.D., Doberstein, S.K., and Zot, H.G. 1991. Myosin-I. Ann. Rev. Physiol., 53: 653-681.

23. Rayment, I., Holden, H.M., Whittaker, M., Yohn, C.B., Lorenz, M., Holmes, K.C., and Milligan, R.A. 1993. Structure of the actin-myosin complex and its implications for muscle contraction. Science (USA), 261: 58-65.

24. Ritchie, M.D., Geeves, M.A., Woodward, S.A., and MansteIn, D.J. 1993. Kinetic characterization of a cytoplasmic myosin motor domain expressed in Dictyostelium discoideum. Proc. Natl. Acad. Sci. USA, 90: 8619-8623.

25. Romberg, L. and Vale, R.D. 1993. Chemomechanical cycle of kinesin differs from that of myosin. Nature (Lond.), 361: 168-170.

26. Titus, M.A., Wessels, D., Spudich, J.A., and Soll, D. 1993. The unconventional myosin encoded by the myoA gene plays a role in Dictyostelium motility. Mol. Biol. Cell, 4: 233- 
246.

27. White, H.D. and TAYloR, E.W. 1976. Energetics and mechanism of actomyosin adenosine triphosphatase. Biochemistry, 15: $5818-5826$.

28. WOLENSKI, J.S. 1995. Regulation of calmodulin-binding myosins. Trends in Cell Biol., 5: 310-316.
29. Yonemura, S. and Pollard, T.D. 1990. Localization of myosin-I and myosin-II in Acanthamoeba. J. Cell Sci., 102: 629642.

30. Zot, H.G., Doberstein, S.K., and Pollard, T.D. 1992. Myosin-I moves actin filaments on a phospholipid substrate: implications for membrane targeting. J. Cell Biol., 116: 367-376. 